\title{
Topical benzoyl peroxide application on the shoulder reduces Propionibacterium acnes: a randomized study
}

Vendela Scheer, Malin Bergman, Maria Lerm, Lena Serrander and Anders Kalén

The self-archived postprint version of this journal article is available at Linköping University Institutional Repository (DiVA):

http:/ / urn.kb.se/ resolve?urn=urn:nbn:se:liu:diva- 148372

N.B.: When citing this work, cite the original publication.

Scheer, V., Bergman, M., Lerm, M., Serrander, L., Kalén, A., (2018), Topical benzoyl peroxide application on the shoulder reduces Propionibacterium acnes: a randomized study, J ournal of shoulder and elbow surgery, 27(6), 957-961. https:/ / doi.org/ 10.1016/j.jse.2018.02.038

Original publication available at:

https:/ / doi.org/ 10.1016/j.jse.2018.02.038

Copyright: Elsevier

http:/ / www.elsevier.com/

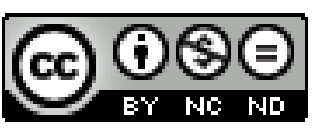


1 Topical benzoyl peroxide application on the shoulder reduces Propionibacterium acnes;

2 a randomized study

3

4 Running head

$5 \quad$ P.acnes reduction with skin preparation

6 Authors

7 Vendela M Scheer Department of Clinical and Experimental Medicine, Linköping

$8 \quad$ University, Linköping, SE 58185 Sweden, vendela.scheer@liu.se

$9 \quad$ Lena Serrander Division of Clinical Microbiology, Department of Clinical and

10 Experimental Medicine, Linköping University, Linköping, SE 581 85, Sweden

11 Maria Lerm Department of Clinical and Experimental Medicine, Linköping University;

12 Malin Berman Jungeström Divison of Clinical Microbiology, Faculty of Health Sciences,

13 University Hospital, Linköping, Sweden

14 Anders Kalen Division of orthopedics, Department of Clinical and Experimental Medicine Faculty of Health Sciences at Linköping University, Linköping SE 58185 Sweden

17 Acknowledgments:

18 Susanne Olivesjö for assisting during the study occasions

Johan Scheer for helping with the manuscript 


\section{P.acnes reduction with skin preparation}

1 Preoperative topical benzoyl peroxide of the shoulder reduces P.acnes and prevents recolonization, compared to chlorhexidine soap.

\section{Abstract}

5 Background: Propionibacterium acnes (P.acnes) is a common cause of infection following

6 shoulder surgery. Studies have shown that standard surgical preparation does not eradicate

$7 \quad$ P.acnes. The purpose of this study was to examine if topical application with benzoyl peroxide gel (BPO) could decrease the presence of P.acnes, compared to the today's standard treatment with chlorhexidine soap (CHS). We also investigated and compared the recolonization of the skin after surgical preparation and draping, between the BPO- and CHStreated groups.

Methods: A single blinded non-surgical study with forty volunteers - twenty-four men and sixteen women were randomized to preoperative topical treatment at home with either $5 \%$ BPO or $4 \%$ CHS in the area of a deltopectoral approach of their left shoulder. Four skin swabs from the area were taken in a standardized manner at different times: Before and after topical treatment, after surgical skin preparation and sterile draping and 120 minutes after draping.

Results: Topical treatment with BPO significantly reduced the presence of P.acnes as CFU on the skin after surgical preparation. P.acnes was found in 1/20 subjects of the BPO group, and 7/20 in the CHS-group ( $p<0.044)$. The results remained after two hours $(p<0.048)$.

Conclusion: Topical preparation with BPO before shoulder surgery may be effective in reducing P.acnes on the skin and prevent recolonization.

Keywords: Propionibacterium acnes; Preoperative shower; Shoulder; Infection; Benzoyl peroxide; Chlorhexidine 
P.acnes reduction with skin preparation

26 Level of evidence: Level II 


\section{P.acnes reduction with skin preparation}

\section{Introduction}

Propionibacterium acnes (P.acnes) is a gram-positive facultative anaerobic rod, a human commensal bacteria who resides in the pilosebaceous ducts of the skin ${ }^{1,6}$. The reported numbers of shoulder infections after surgery caused by $P$. acnes is increasing and so is the incidence of resistance to antibiotics $1,3,11,16,21,26$. The ability of P.acnes to create biofilm causes severe infections that may involve reoperation and long-term antibiotic treatment. To decrease the bacterial burden on the skin before operation one strategy is topical preparation at home with chlorhexidine soap (CHS). Despite strict preoperative preparation with chlorhexidine solution in $70 \%$ ethanol earlier studies has shown that chlorhexidine is not able to eradicate P.acnes from the skin. From $7 \%$ up to $50 \%$ of P.acnes may still be present on the skin ${ }^{10,15,23,25}$. Benzoyl peroxide (BPO) is widely used as topical therapy for acne vulgaris, and has so been for more than five decades. The bactericidal effect of BPO on P.acnes is well documented, and has not been associated with the development of P.acnes resistance. $^{4,8,12,17}$. The purpose of this study was to examine if topical application with BPO could decrease the presence of P.acnes on the treated skin, compared to the today's standard treatment with CHS. We also investigated and compared the recolonization of the skin after surgical preparation and draping, between the BPO- and CHS-treated groups. 


\section{P.acnes reduction with skin preparation}

49

50

\section{Material and Methods}

A single-blinded non-surgical randomized study, with forty healthy volunteers in aged from 20 to 66 , twenty-four men and sixteen women gave informed consent to participate.

Exclusion criteria were antibiotic treatment 10 days prior to trial day, presence of diabetes mellitus, local skin lesions and local or systemic corticoid steroid treatment. Participants were randomized in blocks of four to CHS or BPO-pretreatment. The investigator was blinded to allocated treatment. One week prior to the trial day the participants received verbal and written instructions. Thereafter the first skin swab was collected on the left shoulder (Sample A).

The groups prepared as follows:

1. BPO group

The treatment set up in the BPO-group was designed in collaboration with a dermatologist, who advised on drug concentration and application frequency to minimize local side effects, e.g. erythema, peeling and dryness. Hence the BPO group started the procedure 48 hours before the trial day. After showering and drying they applied a $5 \mathrm{~cm}$ strip of $5 \%$ BPO on the left shoulder. They repeated the application the following morning and evening. The fifth and last time was the morning on trial day.

\section{CHS group}

According to the local routine protocol the CHS group prepared with $4 \%$ chlorhexidine soap on their left shoulder, starting the day before the trial day with two 


\section{P.acnes reduction with skin preparation}

showers, with a minimum of two hours in between, using two sponges each, and on trial day one shower in the morning with two more sponges.

A treatment diary was administered to each participant for affirmation of each gel application or shower, showing $100 \%$ compliance. On each trial day occasion four volunteers were placed on separate beds in the same operating room with laminar airflow (LAF) with their upper body inside the LAF-circle. Before surgical preparation the next skin swab was collected from the left treated side (Sample B). At the same time a control swab was taken from the contralateral shoulder. A skin swab was collected after the treated left side was prepared for 2 minutes with $0.5 \%$ chlorhexidine solution in $70 \%$ ethanol, and sterile drape was applied (Sample C). 120 minutes after surgical preparation and sterile draping the last skin swab was collected (Sample D) (Table 1). All skin swabs were taken by rub 15 times over a $10 \mathrm{~cm}$ deltopectoral interval, and immediately put into the medium. Within thirty minutes the skin swabs were transported to the laboratory, vortexed for $10 \mathrm{sec}$ before cultured on anaerobic blood agar medium without antibiotics and placed in an anaerobic incubator. After five days in the incubator the number of colony forming units (CFU) were counted and divided into five groups according to the numbers of CFUs (Table 2). The bacterial colonies were classified on agar plates by surface characteristics. P.acnes was identified with matrixassisted laser desorption/ionization time-of-flight (MALDI-ToF) mass spectrometry. Analyzes were blinded and performed by the main author. Code was broken after analyzes were done. 


\section{P.acnes reduction with skin preparation}

\section{Statistical Analysis}

97

98 For dichotomous variables we used Fischer's exact test and otherwise Chi-squared test. P-

99 values $<0.05$ was considered being statistically significant.

100 


\section{P.acnes reduction with skin preparation}

101

102

103

104

105

106

107

108

112

113

114

115

116

117

\section{Results}

Before any treatment (sample A), P.acnes was detected in 38/40 subjects, and there was no significant difference in CFU between the groups. In the BPO- group, presence of P.acnes diminished with treatment (Figure 1a) but not in the CHS-group (Figure 1b). After skin preparation (Sample C) we could detect CFU of P. acnes in only 1/20 in the BPO-group compared to $7 / 20$ in the CHS-group ( $p=0.044$, Figure 2). Two hours later, the BPO-group showed a significantly lower P.acnes presence than the CHS-group ( $\mathrm{p}=0.048$, Figure 2 ). There was no significant difference in presence of P.acnes before surgical field preparation (Sample B) and after two hours (Sample D) in the CHS-group (Figure 1b), in contrast to the BPO-group (Figure 1a).

The total number of CFU (which might comprise of more bacterial strains than P.acnes) also diminished after topical BPO-treatment ( $\mathrm{p}$-value 0.035) but not in the CHS-treated group $(p=0.284)$ 


\section{P.acnes reduction with skin preparation}

\section{Discussion}

To the authors knowledge this is the first randomized study which compares topical BPOtreatment to topical CHS-treatment as preoperative preparations. We show that BPOtreatment significantly decreases the presence of P.acnes after preoperative preparation and the result remains after 120 minutes. To our knowledge only one other study have investigated the effect of BPO-treatment on shoulders undergoing surgery and presented a reduction of P.acnes compared to the untreated contralateral shoulder ${ }^{24}$. It is well-known that chlorhexidine does not eradicate P.acnes on the skin after surgical preparation ${ }^{5,10,25}$, which is in concordance with present study.

We detected a very high proportion of detected P.acnes both at the investigated shoulder $(38 / 40)$ as well as in the control shoulder (37/40). In other studies on shoulders this detection varies between $42-76 \%^{7,18,23}$. The fact that we used healthy volunteers is hardly a sufficient explanation. Neither gender nor age appear to differ compared to earlier studies. A more likely explanation is the method used. Factors that might effect the results are the swabbed area, the pressure applied on the swab, duration and frequency, which may make comparisons difficult. Skin - treated or even untreated - can be difficult to culture P.acnes from, because of its preference to grow deep into the skin. Therefore, prior to this study, we performed a small pilot study where we compared different methods. The pilot study resulted in the choice of procedure with skin- swab that we used in the present study.

It is sometimes stated that P.acnes is more prevalent in men ${ }^{5,13,19}$ something that was not confirmed in our study. This statement may reside on the indirect observation that deep 


\section{P.acnes reduction with skin preparation}

postoperative infection with P.acnes is more often found in the male population ${ }^{26,29}$, whereas other studies using swabs show no gender difference ${ }^{18,23}$

Dermatological studies indicate that the rapid effect of $\mathrm{BPO}^{4,14}$ makes a two day preparation sufficient in reducing P.acnes also minimizing side effect such as redness, dry and scaly skin. These side effects appear in the beginning ${ }^{14,27}$ of treatment and may be a drawback in general treatment with BPO in conjunction with shoulder surgery.

Interestingly there was no difference in the prevalence of P.acnes in the CHS- group before skin preparation until the swab taken after two hours (Figure 1b), while there was a significant reduction in the BPO-group (Figure 1a). Since a risk factor for surgical site infection is duration of surgery ${ }^{2,22,28}$, it appears troublesome that the surgical field has the same amount of $P$.acnes as an unprepared shoulder.

To decrease confounding by external bacterial seeding, it must be emphasized that the sampling was performed under as surgery like conditions as possible in an operation room with state-of-the-art laminar air flow and sterile draping. Also subject compliance to assigned treatment was $100 \%$.

There are limitations to our study. Using healthy volunteers may not reflect the anticipated response to BPO in patients - presumably older and with co-morbidities - undergoing shoulder surgery. These may have a different bacterial flora and response to BPO. Furthermore one may anticipate that there is a correlation between $P$. acnes on the skin from swabs and in the deeper layers but that has not to our knowledge been shown; reduction of the latter probably being clinically important. A larger study population could have shown 


\section{P.acnes reduction with skin preparation}

differences in the CHS-group, but our findings on this subject are consistent with those of other studies $9,15,20,23,25$. Quantification of colonizing bacteria on the skin pre-operatively has often been used as a marker for risk of post-operative infection, but how well it really corresponds to risk for infections for different bacterial species is not so well studied.

\section{Conclusion}

In summary, this non-surgical case study, shows that there is a significant difference between the BPO and CHS group immediately after surgical preparation and that the results remained after 120 minutes. The skin swabs give micro biotic data of the skin, if that has any correlation to SSI we do not know. The most likely explanation is that BPO affects the reemergence of P.acnes from deeper layers and thereby decreases recolonization of the skin. Given this evidence for the effect of BPO on the skin after surgical preparation and over time, topical preparation with BPO before shoulder surgery may be effective in reducing P.acnes on the skin and prevent recolonization. 


\section{P.acnes reduction with skin preparation}

\section{References}

1 Achermann Y, Goldstein EJ, Coenye T, Shirtliff ME. Propionibacterium acnes: from commensal to opportunistic biofilm-associated implant pathogen. Clin Microbiol Rev 2014;27:419-440. 10.1128/CMR.00092-13

2 Allegranzi B, Zayed B, Bischoff P, Kubilay NZ, de Jonge S, de Vries F et al. New WHO recommendations on intraoperative and postoperative measures for surgical site infection prevention: an evidence-based global perspective. The Lancet Infectious diseases 2016. 10.1016/s1473-3099(16)30402-9

3 Athwal GS, Sperling JW, Rispoli DM, Cofield RH. Deep infection after rotator cuff repair. J Shoulder Elbow Surg 2007;16:306-311. 10.1016/j.jse.2006.05.013

4 Bojar RA, Cunliffe WJ, Holland KT. The short-term treatment of acne vulgaris with benzoyl peroxide: effects on the surface and follicular cutaneous microflora. The British journal of dermatology 1995;132:204-208.

5 Chuang MJ, Jancosko JJ, Mendoza V, Nottage WM. The Incidence of Propionibacterium acnes in Shoulder Arthroscopy. Arthroscopy : the journal of arthroscopic \& related surgery: official publication of the Arthroscopy Association of North America and the International Arthroscopy Association 2015;31:1702-1707. 10.1016/j.arthro.2015.01.029

6 Dessinioti $C$, Katsambas A. Propionibacterium acnes and antimicrobial resistance in acne. Clin Dermatol 2017;35:163-167. 10.1016/j.clindermatol.2016.10.008

7 Dizay HH, Lau DG, Nottage WM. Benzoyl peroxide and clindamycin topical skin preparation decreases Propionibacterium acnes colonization in shoulder arthroscopy. J Shoulder Elbow Surg 2017. 10.1016/j.jse.2017.03.003

8 Dreno B. Topical antibacterial therapy for acne vulgaris. Drugs 2004;64:2389-2397.

9 Falk-Brynhildsen K, Friberg O, Soderquist B, Nilsson UG. Bacterial colonization of the skin following aseptic preoperative preparation and impact of the use of plastic adhesive drapes. Biol Res Nurs 2013;15:242-248. 10.1177/1099800411430381

10 Falk-Brynhildsen K, Soderquist B, Friberg O, Nilsson UG. Bacterial recolonization of the skin and wound contamination during cardiac surgery: a randomized controlled trial of the use of plastic adhesive drape compared with bare skin. The Journal of hospital infection 2013;84:151-158. 10.1016/j.jhin.2013.02.011

11 Hackett DJ, Crosby LA. Infection Prevention in Shoulder Surgery. Bull Hosp Jt Dis (2013) 2015;73:140-144.

12 Kircik LH. The role of benzoyl peroxide in the new treatment paradigm for acne. Journal of drugs in dermatology : JDD 2013;12:s73-76.

13 Koh CK, Marsh JP, Drinkovic D, Walker CG, Poon PC. Propionibacterium acnes in primary shoulder arthroplasty: rates of colonization, patient risk factors, and efficacy of perioperative prophylaxis. J Shoulder Elbow Surg 2015. 10.1016/j.jse.2015.09.033

14 Kosmadaki M, Katsambas A. Topical treatments for acne. Clin Dermatol 2017;35:173-178. 10.1016/j.clindermatol.2016.10.010

15 Lee MJ, Pottinger PS, Butler-Wu S, Bumgarner RE, Russ SM, Matsen FA. Propionibacterium Persists in the Skin Despite Standard Surgical Preparation. The Journal of Bone \& Joint Surgery 2014;96:1447-1450. 10.2106/jbjs.m.01474

16 Leyden J, Levy S. The development of antibiotic resistance in Propionibacterium acnes. Cutis 2001;67:21-24.

17 Leyden JJ, Del Rosso JQ, Webster GF. Clinical considerations in the treatment of acne vulgaris and other inflammatory skin disorders: focus on antibiotic resistance. Cutis 2007;79:9-25.

18 Matsen FA, 3rd, Butler-Wu S, Carofino BC, Jette JL, Bertelsen A, Bumgarner R. Origin of propionibacterium in surgical wounds and evidence-based approach for culturing propionibacterium from surgical sites. The Journal of bone and joint surgery American volume 2013;95:e1811-1817. 10.2106/JBJS.L.01733 


\section{P.acnes reduction with skin preparation}

19 Mook WR, Klement MR, Green CL, Hazen KC, Garrigues GE. The Incidence of Propionibacterium acnes in Open Shoulder Surgery: A Controlled Diagnostic Study. The Journal of bone and joint surgery American volume 2015;97:957-963. 10.2106/jbjs.n.00784

20 Murray MR, Saltzman MD, Gryzlo SM, Terry MA, Woodward CC, Nuber GW. Efficacy of preoperative home use of $2 \%$ chlorhexidine gluconate cloth before shoulder surgery. J Shoulder Elbow Surg 2011;20:928-933. 10.1016/j.jse.2011.02.018

21 Patel A, Calfee RP, Plante M, Fischer SA, Green A. Propionibacterium acnes colonization of the human shoulder. J Shoulder Elbow Surg 2009;18:897-902. 10.1016/j.jse.2009.01.023

22 Peersman G, Laskin R, Davis J, Peterson MG, Richart T. Prolonged operative time correlates with increased infection rate after total knee arthroplasty. HSS J 2006;2:70-72. 10.1007/s11420-005-0130-2

23 Phadnis J, Gordon D, Krishnan J, Bain GI. Frequent isolation of Propionibacterium acnes from the shoulder dermis despite skin preparation and prophylactic antibiotics. J Shoulder Elbow Surg 2016;25:304-310. 10.1016/j.jse.2015.08.002

24 Sabetta JR, Rana VP, Vadasdi KB, Greene RT, Cunningham JG, Miller SR et al. Efficacy of topical benzoyl peroxide on the reduction of Propionibacterium acnes during shoulder surgery. Journal of Shoulder and Elbow Surgery 2015;24:995-1004. 10.1016/j.jse.2015.04.003

25 Saltzman MD, Nuber GW, Gryzlo SM, Marecek GS, Koh JL. Efficacy of surgical preparation solutions in shoulder surgery. The Journal of bone and joint surgery American volume 2009;91:1949-1953. 10.2106/jbjs.h.00768

26 Singh JA, Sperling JW, Schleck C, Harmsen WS, Cofield RH. Periprosthetic infections after total shoulder arthroplasty: a 33-year perspective. J Shoulder Elbow Surg 2012;21:15341541. 10.1016/j.jse.2012.01.006

27 Sittart JA, Costa A, Mulinari-Brenner F, Follador I, Azulay-Abulafia L, Castro LC. Multicenter study for efficacy and safety evaluation of a fixeddose combination gel with adapalen $0.1 \%$ and benzoyl peroxide 2.5\% (Epiduo(R) for the treatment of acne vulgaris in Brazilian population. Anais brasileiros de dermatologia 2015;90:1-16. 10.1590/abd18064841.20153969

28 Urquhart DM, Hanna FS, Brennan SL, Wluka AE, Leder K, Cameron PA et al. Incidence and risk factors for deep surgical site infection after primary total hip arthroplasty: a systematic review. J Arthroplasty 2010;25:1216-1222 e1211-1213. 10.1016/j.arth.2009.08.011

29 Wang B, Toye B, Desjardins M, Lapner P, Lee C. A 7-year retrospective review from 2005 to 2011 of Propionibacterium acnes shoulder infections in Ottawa, Ontario, Canada. Diagnostic microbiology and infectious disease 2013;75:195-199. 10.1016/j.diagmicrobio.2012.10.018 


\section{P.acnes reduction with skin preparation}

\section{Figure legends}

271

272 Figure 1. Skin swabs were analyzed from the treated left shoulder and presence of P.acnes

273 (yes or no) was detected. 1a: BPO-group $(n=20)$. 1b: CHS-group $(n=20)$. Time of sampling, 274 see Table 1.

$275 *$ statistically significant.

276

277 Figure 2 Skin swabs were analyzed from left shoulder and presence of P.acnes (yes or no)

278 was detected in BPO -treated group $n=20$, compared with CHS-treated group, $n=20$. Time

279 of sampling, see Table 1.

280

*statistically significant.

281

282 
1a. BPO group

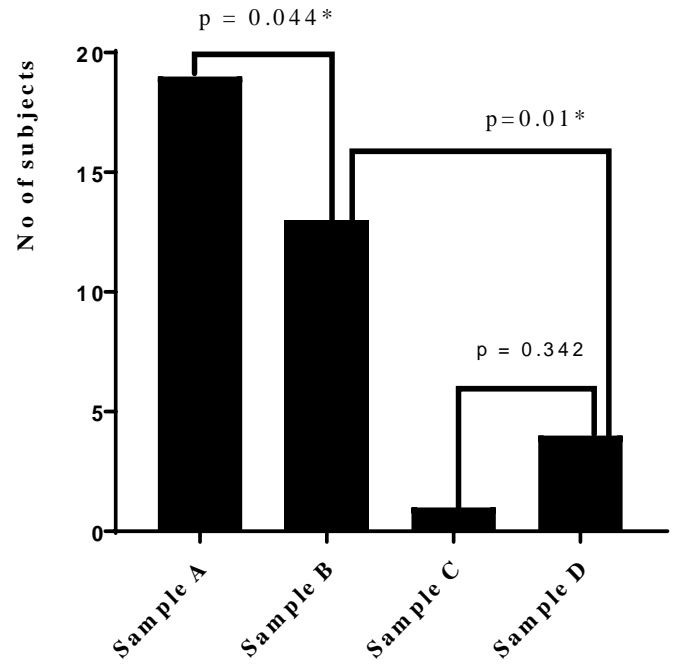

1b. CH S group

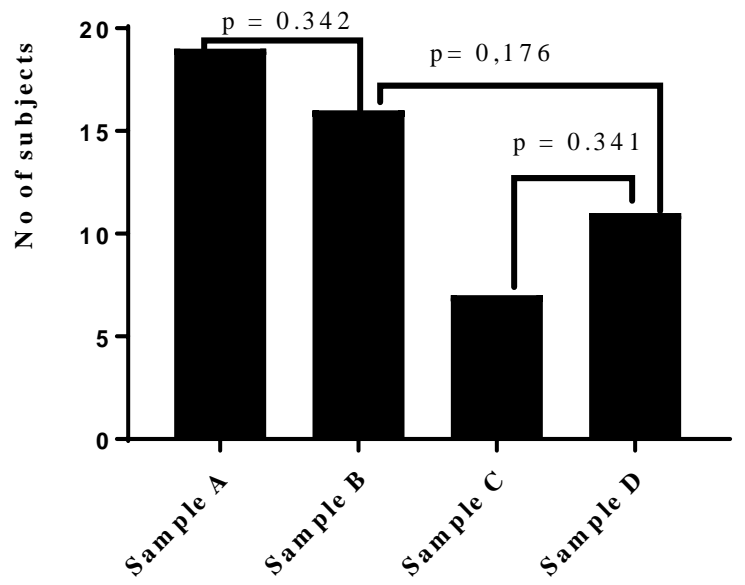


2. Presence of P.acnes between groups

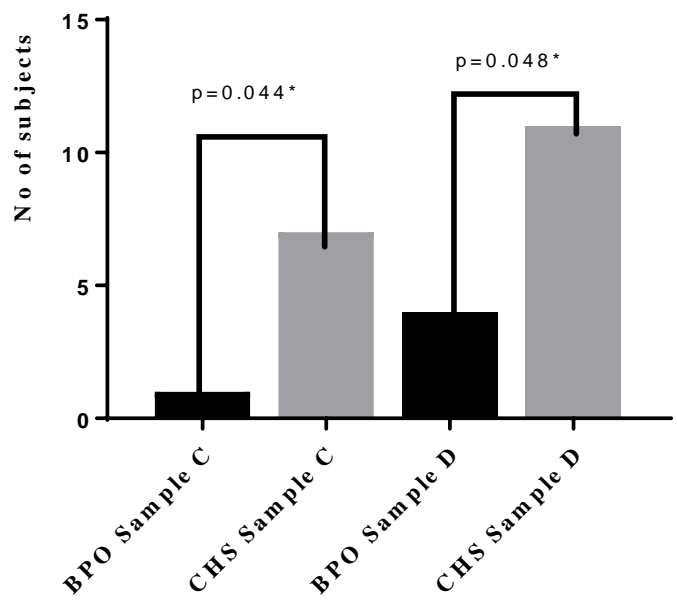




\section{P.acnes reduction with skin preparation}

Table 1. Flow chart of skin swab

\begin{tabular}{|l|l|}
\hline Sample & Time \\
\hline A & Before treatment, one week before trial day \\
\hline B & Trial day, after topical treatment at home \\
\hline C & After surgical preparation and sterile draping \\
\hline D & 120 min after surgical preparation and sterile drape \\
\hline Control & Trial day, right shoulder, not treated \\
\hline
\end{tabular}


Table 2. Grouping of colony forming units (CFU).

\begin{tabular}{|c|c|c|c|c|c|}
\hline Group & 0 & 1 & 2 & 3 & 4 \\
\hline CFU & 0 & $1-15$ & $16-100$ & $>100<1000$ & $>1000$ \\
\hline
\end{tabular}

